\title{
Isokinetic Dynamometry and 1RM Tests Produce Conflicting Results for Assessing Alterations in Muscle Strength
}

\author{
by \\ Paulo Gentil1, Fabricio Boscolo Del Vecchio², Antonio Paoli ${ }^{3}$ Brad J Schoenfeld 4 , \\ Martim Bottaro ${ }^{5}$
}

The purpose of this study was to compare strength gains in the lower limbs, assessed by one maximum repetition (1RM) and isokinetic peak torque (PT), in young men undergoing a resistance training (RT) program. Twenty-seven young men performed resistance training twice a week for 11 weeks. Training involved two exercises for the lower body, two for the upper body and one for the midsection performed with three sets of 8-12 repetitions to momentary muscle failure. Before and after the training period, participants performed the $1 R M$ test in the $45^{\circ}$ leg press and knee extension PT in isokinetic dynamometry. The Pearson correlation coefficient was used to assess the relationship between the changes in IRM and PT, and the Bland-Altman test was performed to check for agreement between the strength changes of both tests. There were significant changes in 1RM and PT of 23.98\% and 15.96\%, respectively $(p<0.05)$. The changes in leg press $1 R M$ were significantly higher than the ones in PT. The Bland-Altman analysis revealed that the tests were not equivalent. In conclusion, professionals and researchers involved in strength assessment should be aware that the results obtained by PT and 1RM are not equivalent when evaluating individual responsiveness and/or the efficacy of an intervention on muscle strength, as the results obtained show large variations and can be even conflicting.

Key words: knee extensors, lower body strength, resistance training.

\section{Introduction}

Muscle strength is an important physical attribute for sports performance (Delecluse, 1997; Jung, 2003; Kraemer et al., 2002) as well as health and longevity (Artero et al., 2012; Newman et al., 2006; Ruiz et al., 2008). Professionals and researchers interested in evaluating muscle strength typically use standardized tests in order to ensure correct measurement and enable the generalization of results (Brown et al., 2001). Two of the most popular tests are the one-repetition maximum (1RM) and the isokinetic peak torque
(PT) (Brown, 2000; Brown et al., 2001). The 1RM test provides the maximum load with which an individual can perform a complete repetition of a given exercise, while the PT test provides the highest torque exerted by the muscles involved in the movement at a constant velocity (Brown et al., 2001). Although the two methods are widely used and accepted by the scientific community, the results obtained from them seem to differ considerably from each other. It is possible to find studies using the 1RM test showing strength gains

\footnotetext{
1 - College of Physical Education and Dance, Federal University of Goias, Goiania, GO, Brazil.

2 - Superior School of Physical Education, Federal University of Pelotas, Pelotas, RS, Brazil.

3 - Department of Biomedical Sciences, University of Padova, Padova, Italy.

4 - Department of Health Sciences, CUNY Lehman College, Bronx, NY, Unites States of America.

5 - College of Physical Education, University of Brasília, Brasília, DF, Brazil.
} 
of more than $100 \%$ after a relatively short period of training (Fiatarone et al., 1994; Frontera et al., 1988), whereas the mean changes measured by the PT test are typically below $20 \%$ after a similar period (de Souza et al., 2010; Feiereisen et al., 2010; Gentil et al., 2010).

Besides the apparent differences in the results obtained from the tests, both are interchangeably used for evaluating muscle strength. However, it is important to test their equivalency before making conclusions concerning the results of a given intervention, as its magnitude can be dependent of the method, such that the results may not be reflective of the intervention, but rather of the tests performed.

Individual variations in muscle strength responsiveness to a given intervention have been a topic of increasing interest, with studies usually classifying individuals according to the magnitude of their alterations in muscle strength (Chmelo et al., 2015; Churchward-Venne et al., 2015; Gentil et al., 2015a). However, if the tests used to evaluate muscle strength are not equivalent, this classification can be biased, since an individual submitted to the same intervention can be classified either as a low or highresponder, depending of the test performed. Therefore, comparing the results obtained by different tests would bring important information for professionals and researchers involved with interventions that have muscle strength as an outcome.

It is important to note that using a correlation coefficient to check for agreement between the tests can be misleading, since a high correlation does not necessarily mean that the two methods agree (Bland et al., 1986). In this regard, the Blood and Altman test may offer a valuable tool for such analysis, as previously proposed (Bland et al., 1986). Although the agreement between strength measures seems to be a basic question to be asked, we were not able to find any studies directly addressing this issue. Therefore, the purpose of this study was to compare strength gains assessed by leg press 1RM and knee extensors PT tests in young men undergoing resistance training.

\section{Material and Methods}

\section{Experimental approach}

The participants performed 11 week resistance training, conducted twice a week, with a minimum interval of 48 hours between following sessions. Initially, there were four familiarization sessions to acquaint participants with the exercises and the training protocol. After the familiarization period, participants were tested for $1 \mathrm{RM}$ in the $45^{\circ}$ leg press and had the PT of knee extensors measured by an isokinetic dynamometer. Tests were performed with 1 to 3 days of rest between them in a randomized order. To decrease the learning effects, the training sessions alternated sled $45^{\circ}$ leg press, seated leg press and knee extension, with only one exercise performed in each training session.

\section{Participants}

An a priori power analysis estimated that a sample size of 23 participants would provide a correlation greater than 0.50 between the tests with statistical power of at least 0.80 at an $\alpha$ level of 0.05 ; however, considering the possibility of experimental attrition during data collection, forty volunteers initially participated in the study. Subjects were recruited through flyers posted at the university and word of mouth. Inclusion criteria required that subjects had no previous experience with resistance training, were at least 18 years of age and were free from any health problems that could be aggravated by the experimental protocol, such as orthopedic and cardiovascular problems. During the study period, participants were instructed not to change their eating habits and not to perform other physical activities beyond the experimental protocol. The data of the participants were included in the analysis only if the attendance was higher than $80 \%$ of the total training sessions (Gentil et al., 2013). Due to low attendance or poor adherence to protocol recommendations, 13 subjects were excluded from the study, leaving 27 participants for final analyses.

All participants were informed verbally and in a written form of all study procedures before providing informed consent. The study was approved by the Local Ethics Committee of the College of Health Sciences at the University of Brasilia.

\section{Procedures}

One-repetition maximum test (1RM)

One week before the beginning of the training period and five to seven days after the last session, the $1 \mathrm{RM}$ load on the sled $45^{\circ}$ leg press 
(Gervasport@, Cotia, Brazil) was determined for each participant. The 1RM protocol followed previous guidelines concerning muscular strength evaluation (Brown et al., 2001). Participants performed a warm-up for two sets of 10 reps at $20-30 \%$ of body weight with 3-min intervals between sets. Three minutes after the warm-up, the load was increased and the participant was instructed to perform a complete repetition. If the repetition was completed successfully, the load was increased by 4 to 20 kilograms and, after a 5min interval, the next repetition was performed. The procedure was repeated until achieving the highest load with which the individual could perform a complete repetition. A maximum of 5 attempts was allowed per session. If the maximum load was not obtained until the fifth attempt, the test was stopped and retaken on the next day. The tests were repeated by all subjects and value of the intraclass coefficient of correlation (ICC) in the present study was 0.96 . We opted to perform the tests in the sled $45^{\circ}$ leg press to provide a real world setting, since it is widely used in experiments and commonly available in gyms and fitness centers.

Peak torque test performed with an isokinetic dynamometer (PT)

The PT of the knee extensors was measured on the right leg with a Biodex system 3 isokinetic dynamometer (Biodex Medical ${ }^{\mathrm{TM}}$, Inc., Shirley, NY). The dynamometer's calibration was performed according to the manufacturer's specifications prior to each test. The subject sat with the dynamometer arm rotation axis aligned with the lateral condyle of the right knee. Belts were used to secure the thigh, pelvis and trunk to the dynamometer chair and prevent uncontrolled movements. The settings and adjustments of the equipment were recorded to ensure that all assessments were performed in the same position. The tests involved two sets of four repetitions at $60 \% / \mathrm{s}$, with 1-min rest intervals between following sets. During the recovery period, the belt at the knees was loosened to allow proper circulation in the lower limbs. The subjects were instructed to fully bend and extend the knee joint and work with maximum intensity during all repetitions. Vigorous verbal encouragement was provided during testing to help ensure best performance. All tests were carried out by the same investigator (Brown, 2000). The test and retest ICC to the PT of knee extensors was 0.98 .

Resistance training

Exercise specialists and exercise technology students supervised all training sessions to ensure participants' safety and adherence to the procedures, in a ratio of 1 supervisor for 5 participants (Gentil et al., 2010). The training protocol consisted of 5 exercises for the major muscle groups: 2 for the lower body (knee extension/seated leg press/sled $45^{\circ}$ leg press and lying leg curl; Figure 1), 2 for the upper body (bench press and lat pull-downs), and 1 for the abdominal muscles (crunches). Participants performed 2 sets of 8-12 repetitions until momentary muscle failure with $2 \mathrm{~min}$ of rest between sets and exercises. To maintain performance in the target repetition range, the loads were reduced if a subject was unable to perform at least 8 repetitions and they were increased if it was possible to perform more than 12 repetitions. The research assistants maintained a training diary for each participant to control loads and repetitions performed during each training session.

Training was carried out twice per week, with a minimum of 48 hours between sessions. Three different types of exercises involving knee extensors were performed (Figure 1): knee extension, seated leg press and sled $45^{\circ}$ leg press (GervaSport ${ }^{\circledR}$, Cotia, Brazil). In each training session, participants performed only one of these exercises in the following order: knee extension $\rightarrow$ seated leg press $\rightarrow$ sled $45^{\circ}$ leg press. Thus, each exercise was performed every 3 sessions. The distribution of exercises was controlled to ensure equivalence in the number of sessions including each exercise. The sled $45^{\circ}$ leg press equipment used during training sessions was also used for $1 R M$ tests. Although the isokinetic test was performed with a device different from the one used for training knee extensions, this exercise was included because it was the closest exercise to the movement performed during the PT tests.

\section{Statistical analysis}

Values are reported as means \pm standard deviation. Data normality was verified by the Kolmogorov-Sminov test. Paired t-tests were used to compare the results of $1 \mathrm{RM}$ and PT tests pre and post training intervention. The PT and 1RM gains were calculated as a percent increase. Effect sizes were calculated using the equation proposed 
by Rhea (Rhea, 2004). The Pearson correlation coefficient was used to assess the relationship between the pre-training values and changes in 1RM and PT. The coefficient of determination $\left(\mathrm{r}^{2}\right)$ was utilized to indicate the percentage of the total variance shared between PT and 1RM changes. A Bland-Altman test was performed to check for agreement between the strength changes as well as between the baseline muscle strength measured by the tests (Bland et al., 1986). Cluster analysis was used to create homogenous groups of low and high responders according to alterations in 1RM and PT, and Pearson chi-square was used to analyze the relationship between the distribution of changes in 1RM and PT.

Results were considered significant when $p<0.05$. The analysis was performed using the Statistical Package for the Social Sciences 17.0 (SPSS, Chicago, IL).

\section{Results}

The mean values of the variables analyzed are presented in Table 1. Mean changes in the sled $45^{\circ}$ leg press $1 \mathrm{RM}$ and knee extensors PT were $23.98 \%(\mathrm{t} 2,27=8,87 ; p<0.001)$ and $15.96 \%(\mathrm{t} 2,27=$ $10.52 ; p<0.001)$, respectively. Comparison between 1RM gains and PT revealed that 1RM increases were significantly higher than changes in PT $(\mathrm{t} 2,27=3.36 ; p=0.02)$. Post hoc analysis revealed that the present study had statistical power greater than 0.99 for detecting changes in leg press 1RM and isokinetic PT.

There was a moderate correlation between baseline PT and leg press 1RM $(r=0.6 ; p$ $<0.01$ ), but the results of Bland-Altman analysis revealed that the measures were not equivalent. The correlation between increases in PT and 1RM were also moderate $(\mathrm{r}=0.49 ; p<0.01$; Figure 2$)$; however, the results of the Bland-Altman tests revealed that the strength gains measured with the two tests were different from each other (Figure 3). The coefficient of determination indicated that the percentage of the total variance shared by the tests was $\mathrm{r}^{2}=0.24(p<0.05)$.

The two clusters for PT alterations were high responders $(n=12): 24.34 \pm 9.4 \%$ and low responders $(n=15): 8.73 \pm 3.54 \%$. The increases in PT were significant for high responders $(p<0.05)$ but not for low responders $(p>0.05)$. The clusters formed for 1RM were: high responders $(n=16)$ : $34.35 \pm 7.83 \%$ and low-responders $(n=11): 11.18 \pm$ $7.3 \%$. Both groups had significant increases in $1 R M(p<0.05)$. According to the results, there was no significant relationship between the distribution of 1RM and PT clusters $(p>0.05)$. This is illustrated in Figure 4, which shows the results obtained in both tests for each participant.

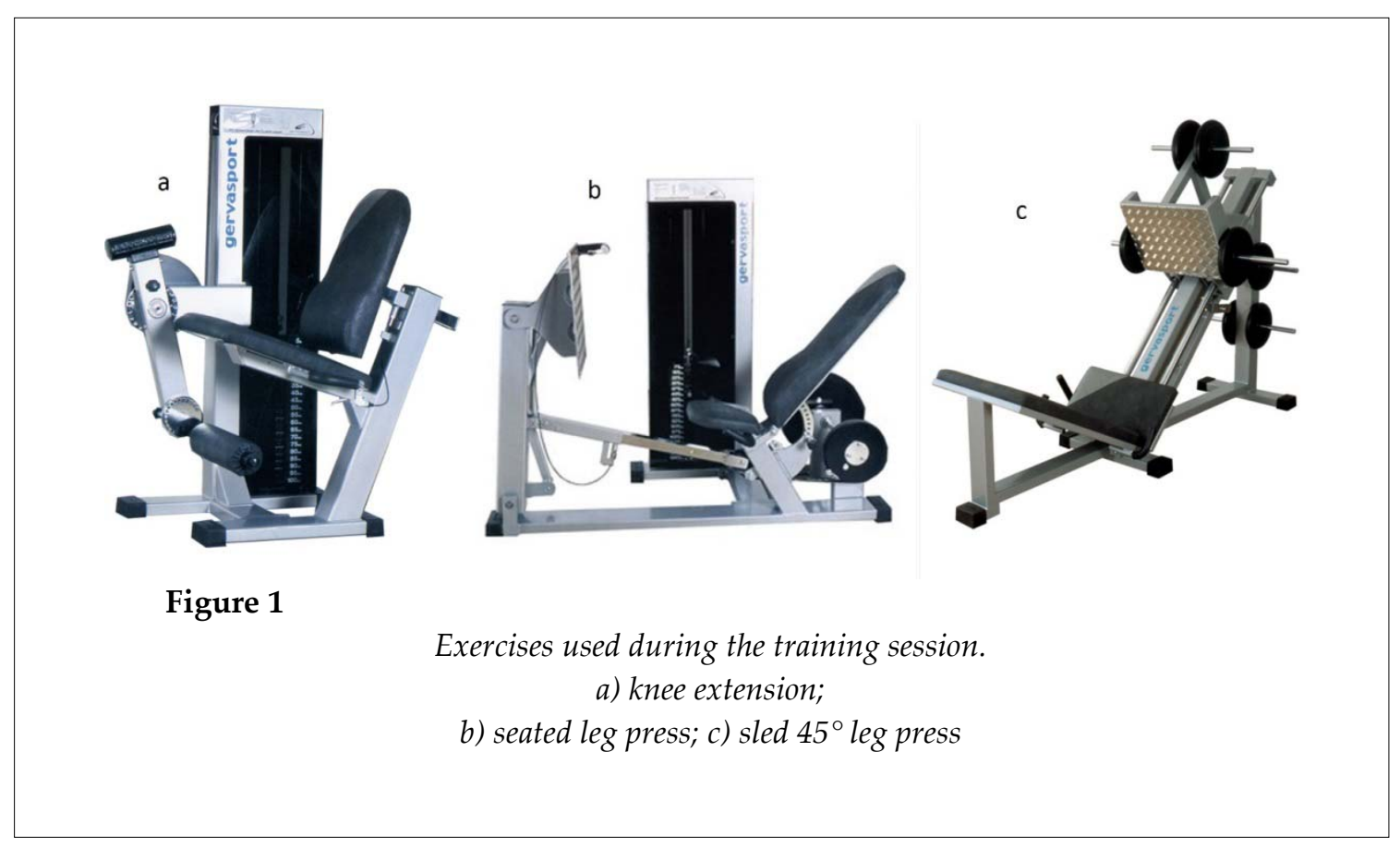



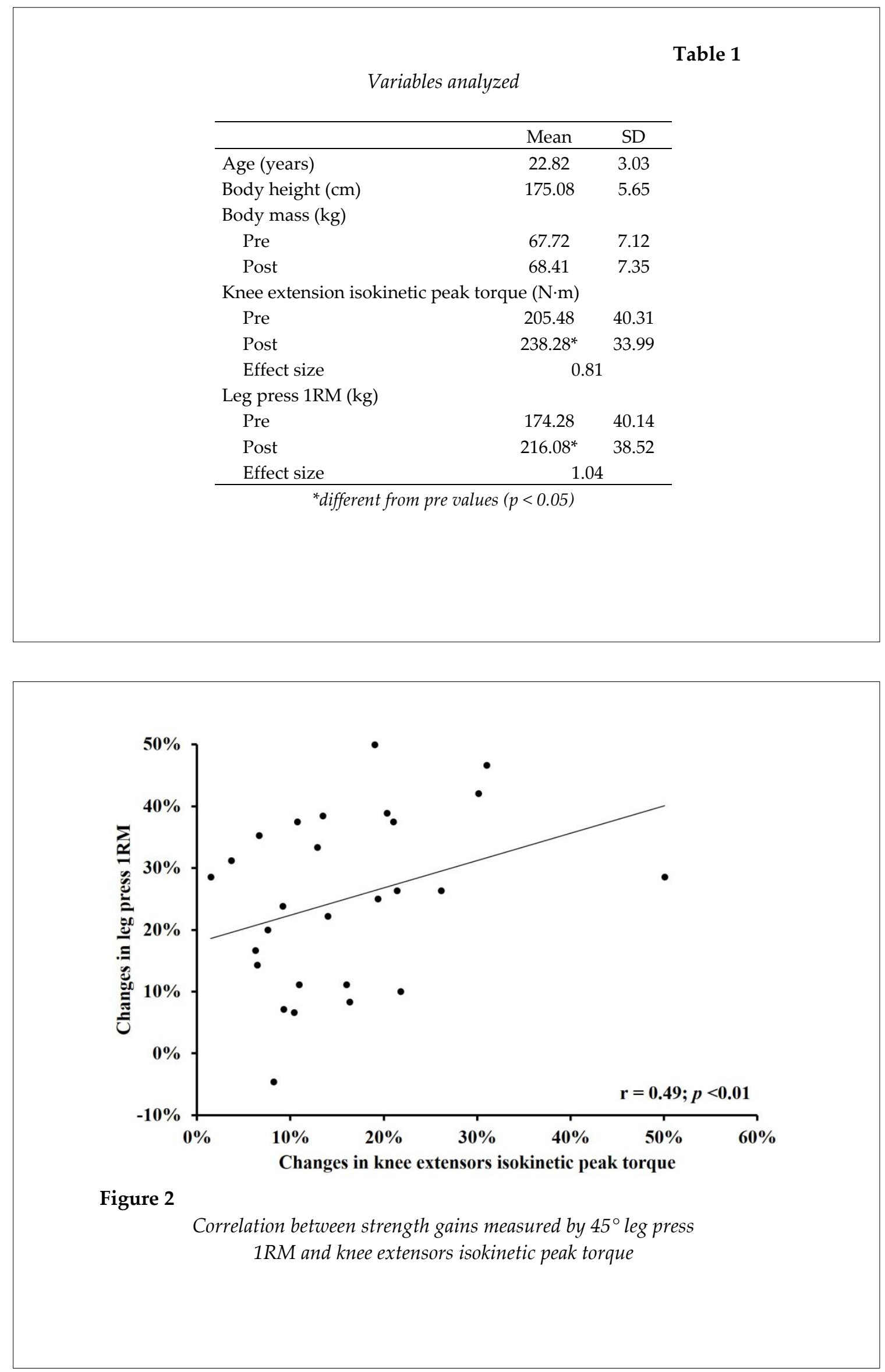


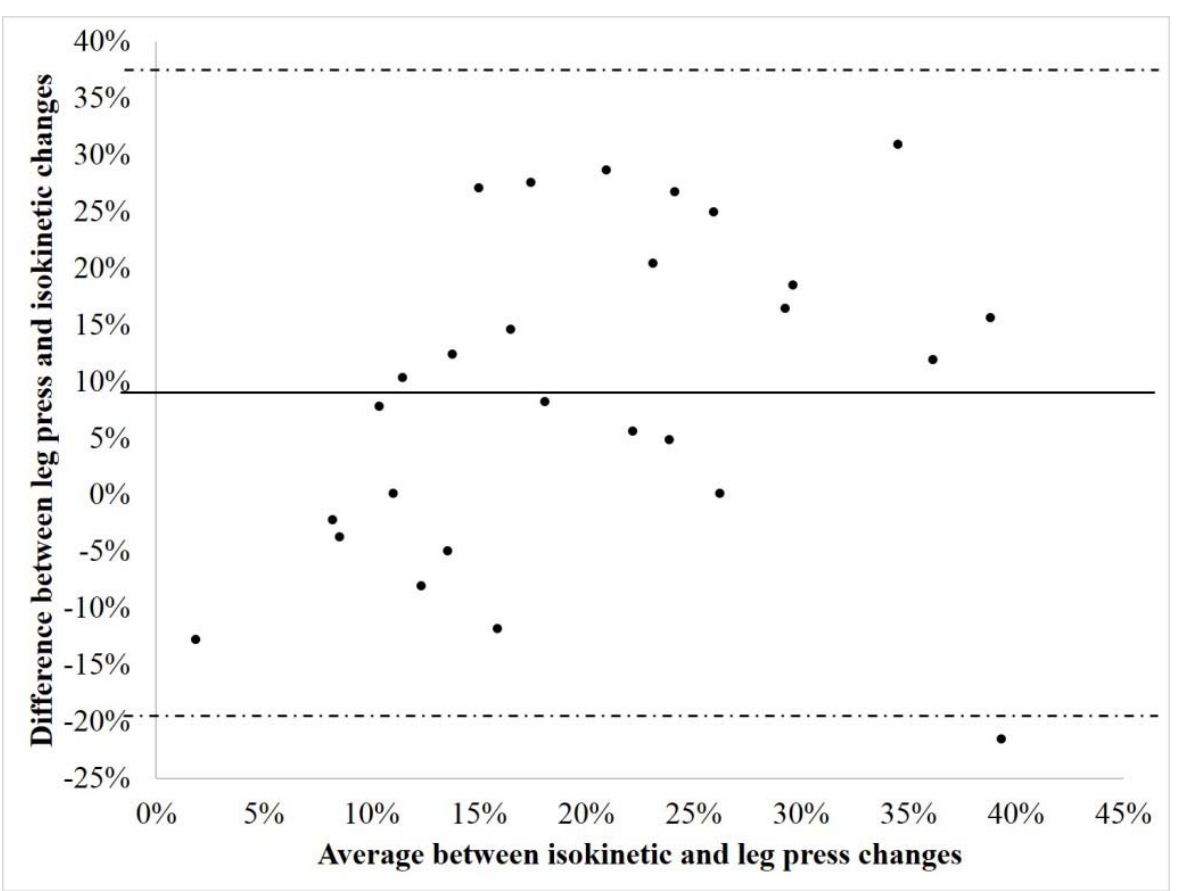

Figure 3

Bland Altman plot between changes in knee extensors isokinetic peak torque and $1 R M$ in the sled $45^{\circ} \mathrm{leg}$ press

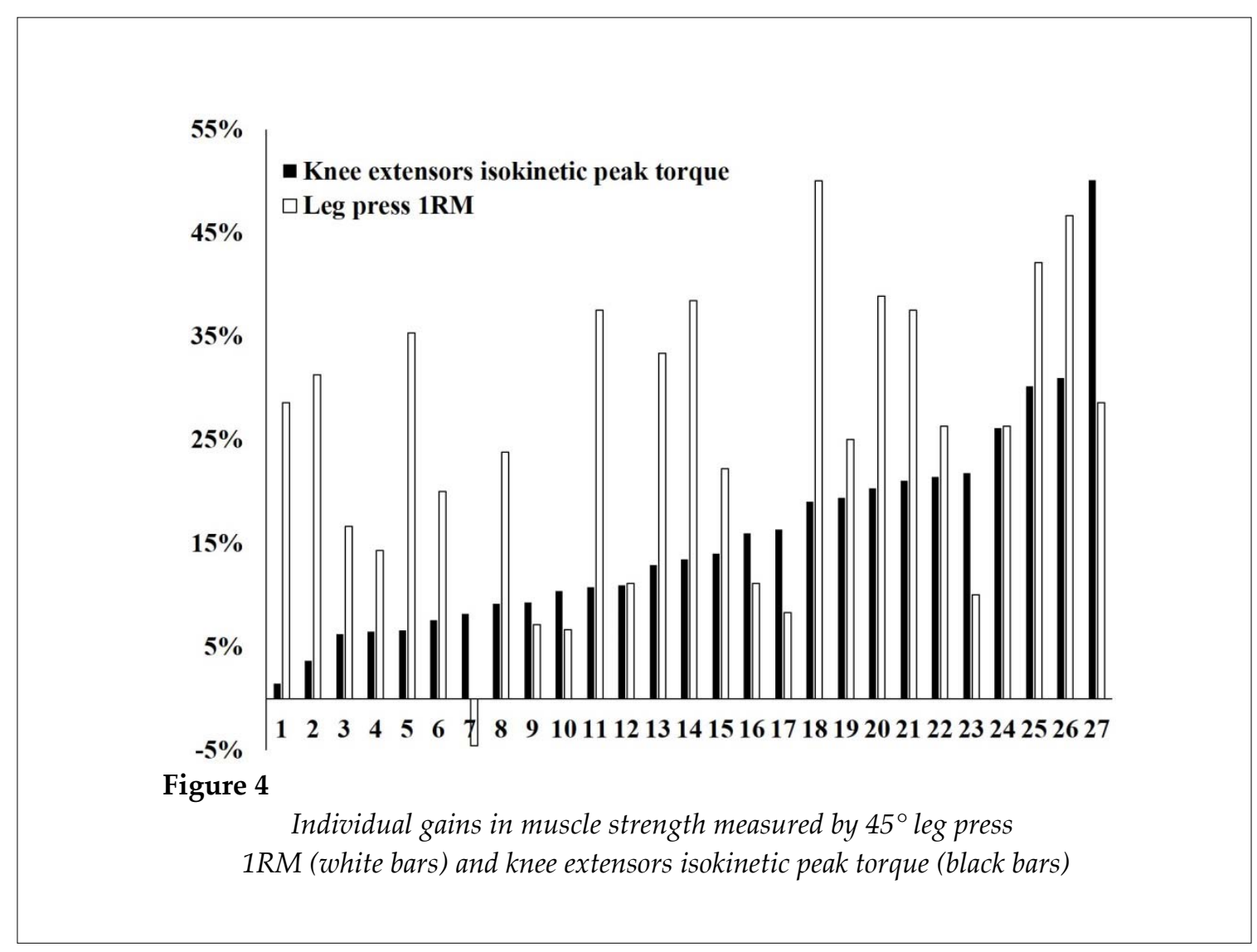




\section{Discussion}

The purpose of the present study was to investigate the relationship between strength gains as measured by two of the most popular methods for assessing muscle strength. After 11 weeks of resistance training, we found a moderate correlation of 0.49 between the results obtained in the $1 \mathrm{RM}$ test in the $45^{\mathrm{o}}$ leg press and the unilateral knee extension PT. The coefficient of determination suggested that $24 \%$ of the variance was shared between the tests. However, the results of the Bland-Altman test revealed that the two measures were not equivalent, which confirms previous suggestion that a significant correlation does not necessarily mean that two methods are equivalent (Bland et al., 1986).

These results occurred even though we attempted to minimize the effects of familiarization by using variations in the exercise performed. One possible explanation is that, despite exercise variations, the participants performed 2 forms of the leg press: seated leg press and sled $45^{\circ}$ leg press, and only 1 exercise resembled the movement performed during the PT test. This is aggravated by the fact that performance of knee extension exercises markedly differs from isokinetic tests. Although both require similar movements, the devices differ considerably in terms of adjustments and the body position and this may have a large impact on results (Murphy et al., 1997). Moreover, knee extensions were conducted using bilateral movements, while the isokinetic test was performed unilaterally. Unilateral and bilateral movements impose different neural requirements, what raises the possibility of specific adaptations (Hakkinen et al., 1996; Taniguchi, 1997). Finally, isokinetic testing was carried out at a constant velocity whereby the subject could produce maximum power at all angles, while training was carried out with isoinertial exercise performed against constant resistance.

In the present study, changes in $1 \mathrm{RM}$ in the $45^{\circ}$ leg press were higher than changes in PT, corroborating previous findings where strength changes evaluated by 1RM testing were higher than the values obtained from PT tests (Abernethy et al., 1996; Feiereisen et al., 2010; Frontera et al., 1988). One may argue that the leg press exercises did not provide enough stimuli for the knee extensors due to its multi joint characteristic. If this is the case, the performance of two sessions involving leg presses and only one with isolated knee extension exercises may have resulted in suboptimal stimuli to the knee extensors muscles, resulting in lower knee extensors PT gains. However, this is improbable, since previous research found that quadriceps muscle activation was similar during leg press and knee extension exercises (Wilk et al., 1996). Moreover, previous studies found that there were no differences in muscle activity (Signorile et al., 1994; Welsch et al., 2005) and muscle strength gains (Gentil et al., $2015 b$ ) when multi joint exercises were compared to single joint ones.

Using cluster analysis, we found that responsiveness to strength training was unequally distributed when strength gains were evaluated by leg press 1RM and isokinetic PT. It is interesting to note that an individual can be classified as a low-responder when changes in muscle strength are analyzed by PT, but as a highresponder when the $1 R M$ is analyzed, and viceversa (as illustrated in Figure 4). The divergent classification in responsiveness between PT and $1 \mathrm{RM}$ occurred in almost $40 \%$ (10 out of the 27 ) of the participants. This is particularly interesting because the heterogeneity of muscle strength response has been a topic of increasing interest (Chmelo et al., 2015; Churchward-Venne et al., 2015; Gentil et al., 2015a). Based on the present results, the classification of an individual as a lowor high-responder can be biased by the test used.

The results of Bland-Altman tests revealed that the tests were not equivalent. This implies that their results cannot be analyzed in the same perspective and should not be taken as if they measured the same outcomes. Therefore, caution should be taken when making conclusions concerning changes in muscle strength from a given intervention, as its magnitude can be dependent on the methods used for strength assessment. For example, one may conclude that an intervention is highly efficient when measuring its results by $1 \mathrm{RM}$, while classifying the results of another intervention as disappointing when evaluating it by isokinetic dynamometry. However, this may not be reflective of the protocols, but rather of the tests performed.

Isokinetic dynamometry and 1RM tests are both considered as valid and often 
interchangeable methods for evaluating changes in muscle strength in nutritional and/or exercise interventions. However, the results of the present study show that their results are not equivalent. Professionals and researchers should be careful when classifying an individual as a low- or highresponder, and be cautious when comparing the results of different exercise and nutritional interventions if muscle strength was evaluated by different tests.

From a practical standpoint, the choice of testing modalities should take into account the desired outcome. If the goal is to determine strength changes over time in a given exercise such as the squat or bench press, then testing should be carried out using the specific exercise. However, if the goal is to evaluate how strength changes from an exercise program promote a general ability to produce force in a given joint, then isokinetic testing should be considered as it provides more neutral determination of transfer of strength.

\section{Acknowledgements}

We would like to thank Eduardo Porto Santos for giving valuable suggestions during manuscript drafting. We would also like to thank Saulo Soares for helping with data collection.

\section{References}

Abernethy PJ, Jurimae J. Cross-sectional and longitudinal uses of isoinertial, isometric, and isokinetic dynamometry. Med Sci Sports Exerc, 1996; 28: 1180-1187

Artero EG, Lee DC, Lavie CJ, Espana-Romero V, Sui X, Church TS, Blair SN. Effects of muscular strength on cardiovascular risk factors and prognosis. J Cardiopulm Rehabil Prev, 2012; 32: 351-358

Bland JM, Altman DG. Statistical methods for assessing agreement between two methods of clinical measurement. Lancet, 1986; 1: 307-310

Brown LE. Isokinetics in Human Performance. Champaing: Human Kinetics; 2000

Brown LE, Weir JP. Procedures recommendation I: accurate assessment of muscular strength and power. J Exerc Physiol, 2001; 4: 1-21

Chmelo EA, Crotts CI, Newman JC, Brinkley TE, Lyles MF, Leng X, Marsh AP, Nicklas BJ. Heterogeneity of physical function responses to exercise training in older adults. J Am Geriatr Soc, 2015; 63: 462-469

Churchward-Venne TA, Tieland M, Verdijk LB, Leenders M, Dirks ML, de Groot LC, van Loon LJ. There Are No Nonresponders to Resistance-Type Exercise Training in Older Men and Women. J Am Med Dir Assoc, 2015; 16: 400-411

de Souza TP, Jr., Fleck SJ, Simao R, Dubas JP, Pereira B, de Brito Pacheco EM, da Silva AC, de Oliveira PR. Comparison between constant and decreasing rest intervals: influence on maximal strength and hypertrophy. J Strength Cond Res, 2010; 24: 1843-1850

Delecluse C. Influence of strength training on sprint running performance. Current findings and implications for training. Sports Med, 1997; 24: 147-156

Feiereisen P, Vaillant M, Eischen D, Delagardelle C. Isokinetic versus one-repetition maximum strength assessment in chronic heart failure. Med Sci Sports Exerc, 2010; 42: 2156-2163

Fiatarone MA, O'Neill EF, Ryan ND, Clements KM, Solares GR, Nelson ME, Roberts SB, Kehayias JJ, Lipsitz LA, Evans WJ. Exercise training and nutritional supplementation for physical frailty in very elderly people. N Engl J Med, 1994; 330: 1769-1775

Frontera WR, Meredith CN, O'Reilly KP, Knuttgen HG, Evans WJ. Strength conditioning in older men: skeletal muscle hypertrophy and improved function. J Appl Physiol, 1988; 64: 1038-1044

Gentil P, Bottaro M. Influence of supervision ratio on muscle adaptations to resistance training in nontrained 
subjects. J Strength Cond Res, 2010; 24: 639-643

Gentil P, Bottaro M. Effects of training attendance on muscle strength of young men after 11 weeks of resistance training. Asian J Sports Med, 2013; 4: 101-106

Gentil P, Ferreira-Junior JB, Bemben MG, Ferreira D, Bottaro M. The Effects of Resistance Training on Lower and Upper Body Strength Gains in Young Women. IJKSS, 2015a; 3: 18-23

Gentil P, Soares S, Bottaro M. Single vs. Multi-Joint Resistance Exercises: Effects on Muscle Strength and Hypertrophy. Asian J Sports Med, 2015b; 6: e24057

Hakkinen K, Kallinen M, Linnamo V, Pastinen UM, Newton RU, Kraemer WJ. Neuromuscular adaptations during bilateral versus unilateral strength training in middle-aged and elderly men and women. Acta Physiol Scand, 1996; 158: 77-88

Jung AP. The impact of resistance training on distance running performance. Sports Med, 2003; 33: 539-552

Kraemer WJ, Ratamess NA, French DN. Resistance training for health and performance. Curr Sports Med Rep, 2002; 1: 165-171

Murphy AJ, Wilson GJ. The ability of tests of muscular function to reflect training-induced changes in performance. J Sports Sci, 1997; 15: 191-200

Newman AB, Kupelian V, Visser M, Simonsick EM, Goodpaster BH, Kritchevsky SB, Tylavsky FA, Rubin $\mathrm{SM}$, Harris TB. Strength, but not muscle mass, is associated with mortality in the health, aging and body composition study cohort. J Gerontol A Biol Sci Med Sci, 2006; 61: 72-77

Rhea MR. Determining the magnitude of treatment effects in strength training research through the use of the effect size. J Strength Cond Res, 2004; 18: 918-920

Ruiz JR, Sui X, Lobelo F, Morrow JR, Jr., Jackson AW, Sjostrom M, Blair SN. Association between muscular strength and mortality in men: prospective cohort study. BMJ, 2008; 337: a439

Signorile JF, Weber B, Roll B, Caruso JF, LOWEN-STEYN I, Perry AC. An electromyographical comparison of the squat and knee extension exercises. J Strength Cond Res, 1994; 8: 178-183

Taniguchi Y. Lateral specificity in resistance training: the effect of bilateral and unilateral training. Eur J Appl Physiol Occup Physiol, 1997; 75: 144-150

Welsch EA, Bird M, Mayhew JL. Electromyographic activity of the pectoralis major and anterior deltoid muscles during three upper-body lifts. J Strength Cond Res, 2005; 19: 449-452

Wilk KE, Escamilla RF, Fleisig GS, Barrentine SW, Andrews JR, Boyd ML. A comparison of tibiofemoral joint forces and electromyographic activity during open and closed kinetic chain exercises. Am J Sports Med, 1996; $24: 518-527$

\section{Corresponding author:}

\section{Paulo Gentil}

FEFD - Faculdade de Educação Física e Dança

Universidade Federal de Goiás - UFG

Campus Samambaia

Avenida Esperança s/n, Campus Samambaia- CEP: 74.690-900

Goiânia - Goiás - Brasil

Phone/Fax: +55 062 3521-1105

Email: paulogentil@hotmail.com 